\title{
Symbiotic star LL Cassiopeiae ${ }^{\star}$ (Research Note)
}

\author{
L. N. Kondratyeva and E. K. Denissyuk
}

\author{
Fessenkov Astrophysical Institute, Observatory 050020 Almaty, Kazakhstan \\ e-mail: kondr@aphi.kz
}

Received 5 December 2006 / Accepted 27 November 2007

\section{ABSTRACT}

\begin{abstract}
Aims. Study of the spectral and photometric properties of the symbiotic star LL Cas. Methods. Spectroscopy and photometry.

Results. Spectral and photometric observations of LL Cas were performed in 1986-2006. LL Cas displays a late-type spectrum with TiO absorbtion bands and emission lines of He II, [O III], He I and H I. Significant fluctuations of relative intensities of [O III], $5007 \AA$ and He II, $4686 \AA$ are revealed. They reflect the changes of the field of radiation of the ionizing source. Wavelike variations of the star's light with amplitudes of about $2^{\mathrm{m}}-3.5$ are found in the $B, V$ and $R$ bands. They can be ascribed to the pulsations of the cool giant.
\end{abstract}

Key words. stars: binaries: symbiotic

\section{Introduction}

LL Cas $\left(\mathrm{PK} 108-035^{\circ} 1=\right.$ PNG $8.3+03.7=$ AN $207.1940=$ PN K4-46) is classified in the General Catalogue of Variable Stars as a Me variable star with a period of 141-days and with $M_{\mathrm{pg}}=15 \cdot 3-17 \mathrm{~m} .0$. Results of infrared observations are presented by Allen (1972). This object was also classified as a planetary nebula K4-46 in the catalog (Perek \& Kohoutek 1967). The spectroscopic and photometric observations of K4-46 (LL Cas) were carried out at the Astrophysical Institute (Kazakhstan) in 1986-2006. Preliminary results were published by Kondratyeva (1992, Paper I). A period of 286.6 days with an amplitude of about 3.5 in the $V$-band was derived.

Some later LL Cas were included in the catalog of Belczinnski et al. (2002) as a symbiotic star with a Mira component. In the present paper the new results of long-term spectroscopic and photometric observations of LL Cas are compiled and analyzed.

\section{Observations}

Spectroscopic observations were performed using a slit spectrograph attached to the 0.7-m telescope of the Astrophysical Institute (Almaty, Kazakhstan). The slit width was 4-5". A three-cascade image tube UM 92 was used as a flux receiver until 1998. Three gratings with different dispersions $\left(1200 \mathrm{~mm}^{-1}\right.$, $600 \mathrm{~mm}^{-1}$ and $400 \mathrm{~mm}^{-1}$ ) and three objective lenses with different spectral response provided a spectral coverage between 3700 and $8600 \AA$, and a reciprocal dispersion in the range of $25-150 \AA \mathrm{mm}^{-1}$ with a spectral resolution of $0.8-10 \AA$. The spectrograms were measured with the microdensitometer using

* Tables 1-3 are only available at the CDS via anonymous ftp to cdsarc.u-strasbg.fr $(130.79 .128 .5)$ or via http://cdsweb.u-strasbg.fr/cgi-bin/qcat?]/A+A/479/529 the density calibrations. Results were then corrected from atmospheric extinction and calibrated from instrumental chromatic response through the observations of standard stars from the catalog (Kharitonov et al. 1988). Wavelength calibration was done using a laboratory source of He I, Ne I and Ar I emission lines. The precision of intensities was about $10-15 \%$ for moderate and strong emission lines. The errors of faint line $(<10$ on the scale of $\mathrm{H} \beta=100$ ) was as high as $50 \%$ or even more. Along with spectrograms, we obtained direct photographs of the object and its environment. The special optical system projected a section of sky about $5^{\prime}$ in diameter onto the photocathode of the image intensifier. The color system, determined mainly by a spectral sensitivity of the photocathode, has a maximum near $5460 \AA$ and a pass band about $800 \AA$, in accordance with Johnson's $V$ band. Some photoelectric observations of LL Cas with broadband filters were carried out by our colleagues $V$. Kardopolov and F. Rspaev on a photoelectric polarimeter mounted on the 1-m Zeiss telescope (Assy-Turgen, Kazakhstan). $V$ magnitudes of four neighboring stars were measured simultaneously. These stars were used as the comparison stars for differential photometry of the object.

In 2002 the spectrograph was equipped with the CCD matrix ST8 $(1530 \times 1020,9 \mu \mathrm{m})$. The modernized variant of the spectrograph provides a dispersion of $0.2-1.5 \AA$ pixel $^{-1}$ in the spectral range 4000-8500 $\AA$. All spectrograms were reduced following the standard procedure consisting of bias subtraction and flat-field normalization, and then all counts were corrected from atmospheric extinction and calibrated from instrumental chromatic response through observations of standard stars from the catalog (Kharitonov et al. 1988). Wavelength calibration was done using a laboratory source of He I, NeI and ArI emission lines. We estimate the errors in the line intensities to be about $10 \%$ for the stronger lines (more than 10 on the scale of $\mathrm{H} \beta=100$ ) and about $15 \%$ for the weaker lines. 


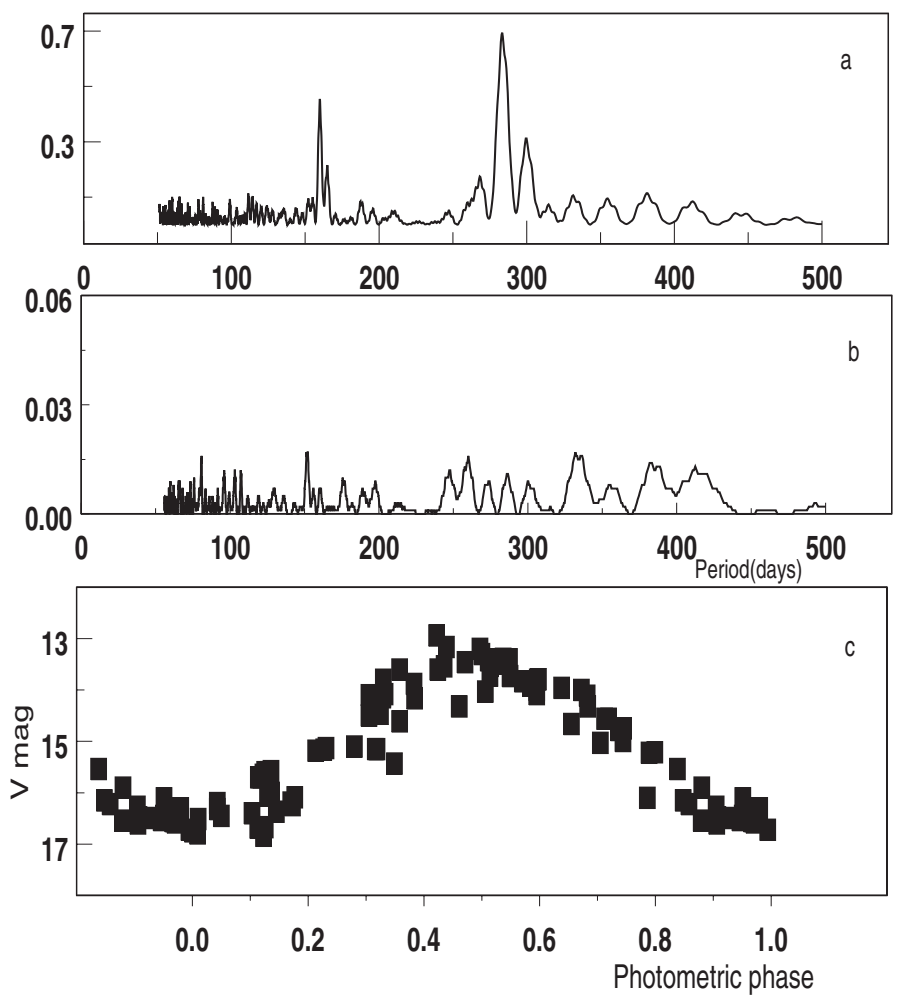

Fig. 1. a) DFT power spectrum of the $V$ data. b) Residuals after the subtraction of a 283.02 days periodicity. c) $V$ light curve folded with a $P=283.02$ days.

Beginning from 2003, photometric observations of LL Cas in the $B, V$ and $R\left(\lambda_{\max }=6600 \AA\right)$ bands were derived with the same CCD camera, attached to the 0.7-m telescope. Some observations were carried out with the 1-m Zeiss telescope. A set of $B, V$ and $R$ filters was used. All frames were dark subtracted and flat fielded. Transformation to the international system was made by measuring the standard stars from Kornilov et al. (1991).

\section{Results}

Table 1 contains the dates of observations, the Julian dates, epochs and the $B, V, R$ magnitudes. The $V$ magnitudes, obtained with the image tube, are accurate to $0.02-0.1$ depending on the brightness of a star. The intrinsic errors of the $B, V$ and $R$, received with CCD, are equal to $0.02-0 \mathrm{~m} 07$ depending on the magnitudes. In the last column of Table 1, the type of observations (spectral or photometric) is given. Data, obtained with the 1.0-meter telescope are denoted by asterisk in the first column.

\subsection{Photometric study of LL Cas}

The very first estimation of a period ( $P=141$ days) with a primary maximum at JD2427957 was made by Huth (1957). Later, the strong periodicity with the ephemeris Max = JD 2427957 $+286.6 \mathrm{E}$ was found in Paper I with the method of Lafler and Kinman.

New analysis of all photometric data taking during 1986-2006 is carried out in this paper. The search for period light variations in the $V$ band has been performed with a discrete Fourier transform (DFT) algorithm. The most prominent peak corresponds to $f=0.00353$ or $P=283.02$ (Fig. 1a). When the main component was subtracted from the data set, no subsequent components were found (Fig. 1b). Thus $P=283^{\mathrm{d}} .02 \pm 1.22$ was accepted as real period modulation. The phase diagram for $V$ data according to the ephemeris $\mathrm{JD}_{\max }=2427957.000+$ $283.02 \times E$ is presented in Fig. 1c.

\subsection{Spectral study of LL Cas}

LL Cas displays a late-type spectrum with the absorption bands and emission line spectrum of an ionized envelope. Some spectral features vary as a function of the light phase. The late type spectrum with the $\mathrm{TiO}$ bands dominates at light maximum, and near the minimum the spectrum of the object looks like a planetary nebula.

Figures 2 and 3 show the reduced spectra of LL Cas for the different photometric phases. The $Y$-axis displays the relative intensities on the scale of $I(\mathrm{H} \beta)=100$. It can be clearly seen, that relative intensities of [O III] and He II lines are exposed to significant changes, and these variations are not correlated with a phase. Recent spectrograms (Fig. 3) show the weak [O III] emission and no He II. Instead, the emission of He I, $6678 \AA$ appears in the "red" region of the spectra. Such a low degree of ionization is observed in LL Cas for the first time. In Table 2 the line ratios of $[\mathrm{O} \mathrm{III}] / \mathrm{H} \beta$ and $\mathrm{He} \mathrm{II} / \mathrm{H} \beta$ are presented together with Julian dates and epochs. Standard precision of intensity lines was discussed above. For this object, however, the precision of measurements may decrease to $25-30 \%$ near the maximum of the star's light, because its continuum becomes extremely nonlinear. Fluctuation of line ratios $I(5007) / I(\mathrm{H} \beta), I(4686) / I(\mathrm{H} \beta)$ do not correlate with a photometric phase (Fig. 4a) and most likely reflect a real change of ionization degree. Equivalent widths $E W(\mathrm{H} \alpha)$ and $E W(\mathrm{H} \beta)$ are listed in Cols. 3 and 4 of Table 2. The inner uncertainty of $E W$ is rather low, about $15-30 \%$, the main source of errors is the level of continuum: it is low near a minimum of a star's light, and nonlinear near a maximum. It was found that $E W(\mathrm{H} \alpha)$ and $E W(\mathrm{H} \beta)$ correlate with the photometric phases (see Fig. 4b). In other words, their variations are mainly caused by change of continuum level. The absolute values of fluxes in $\mathrm{H} \alpha$ and $\mathrm{H} \beta$ were received only during the last years. They are calculated by comparing with the fluxes of the standard stars and are listed in the Table 3 . The precision of $F_{\text {abs }}$ is about $25-30 \%$, and again the main source of errors is the nonlinearity of the continuum. $F_{\text {abs }}(\mathrm{H} \alpha)$ shows a certain tendency to increase with time, but fluctuations of $F_{\text {abs }}(\mathrm{H} \beta)$ do not exceed the uncertainties of data. The value of the absorption coefficient $C(\mathrm{H} \beta)$ was calculated using the line ratios from Table 3 for $\mathrm{Te}=12500 \mathrm{~K}$ and $\mathrm{Ne}=10^{4}-10^{6} \mathrm{~cm}^{-3}$. The new value of $C(\mathrm{H} \beta)=1.58 \pm 0.10$ is essentially above the value $C(\mathrm{H} \beta)=0.26 \pm 0.03$, obtained in 1991 (Paper I).

\section{Conclusions}

The long-term photometric and spectroscopic observations of LL Cas showed the wave-like variability in the $B, V$ and $R$ bands, $(B-V)$ color decrease and $(V-R)$ color increase with decreasing stellar brightness.

The new photometric period 283.02 days was found for all obtained data. In spite of a rather long period of observation, an influence of an orbital movement was not yet recognizable. The plane of an orbit of the symbiotic pair is probably observed under a large angle. Thus we confirm that only Mira pulsations are responsible for the light variations. The emission spectrum of LL Cas is also variable. It was shown that equivalent widths of $\mathrm{HI}$ lines are changed with the photometric phases. However, a large scatter of points (Fig. 4b) and the 

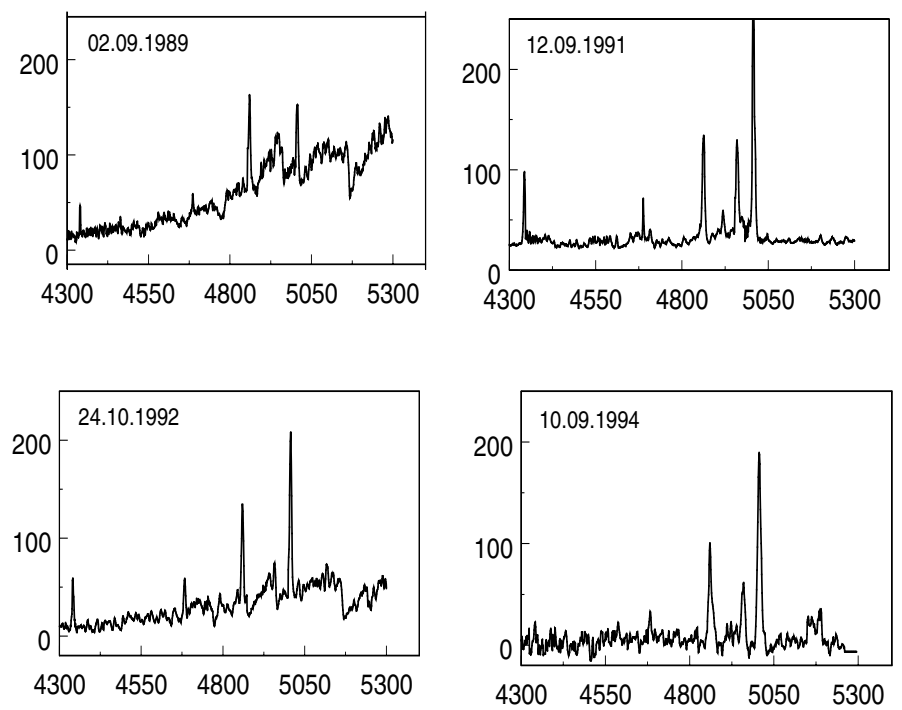

Fig. 2. The blue spectrum of LL Cas in various photometric phases.
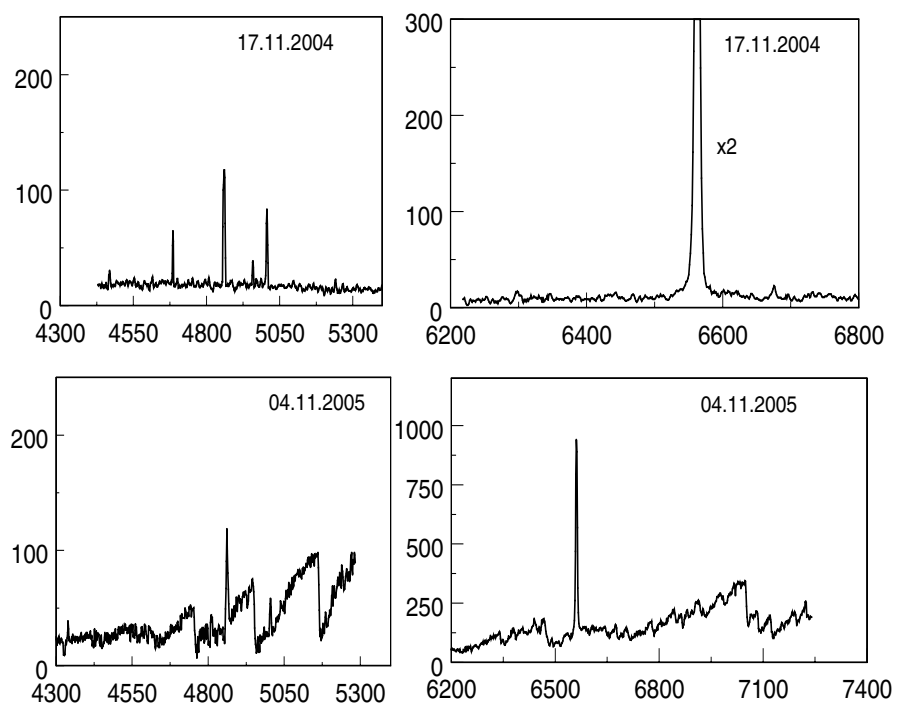

Fig. 3. The recent blue and red spectra of LL Cas.

recent evaluations of $F_{\text {abs }}$ provide evidence that the absolute fluxes of the hydrogen emissions are really being changed with time. Relative intensities of [O III] and He II vary strongly with time. These emissions are formed by different processes, and depend on the physical parameters of a nebula ( $\mathrm{Ne}, \mathrm{Te}$ ) in a different degree. Nevertheless these lines are changing synchronously. Therefore these observable facts probably have a common nature - fluctuations of ionizing radiation. This may
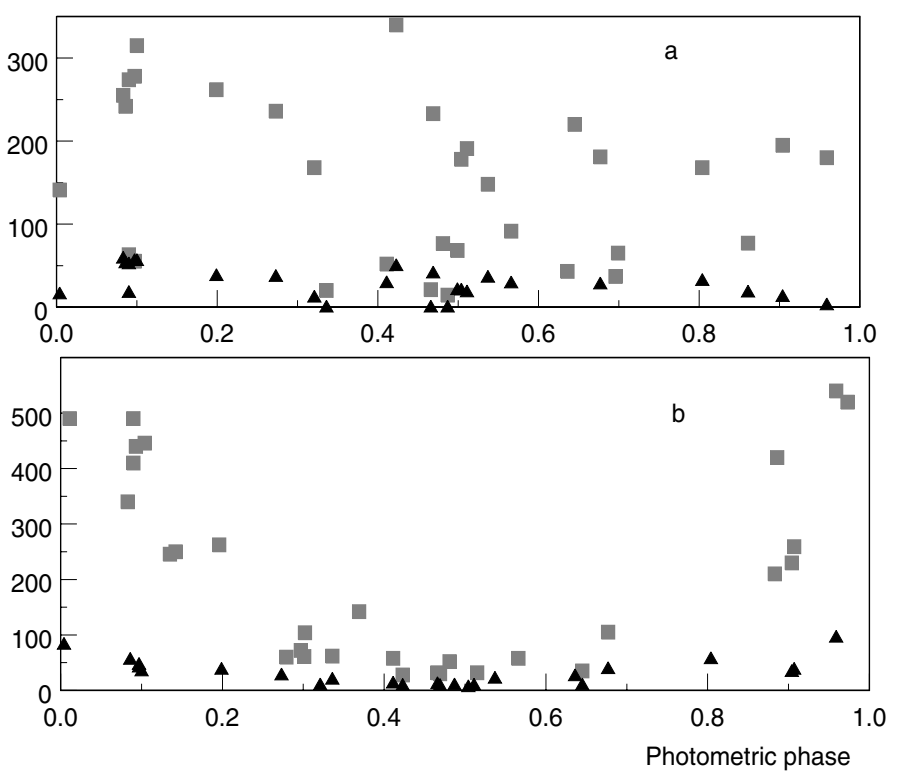

Fig. 4. a) $I(5007)$ (boxes) and $I(4686)$ (triangles) versus a photometric phase. b) $E W(\mathrm{H} \alpha)$ (boxes) and $E W(\mathrm{H} \beta)$ (triangles) versus a photometric phase.

be due to the change of an effective temperature of the hot star or due to the change of absorption of the star's ionizing radiation by the dust component around the star. We used an empirical method by Murset (1994) for estimation of a temperature of the ionizing source. The observable $I P_{\max }=54.4 \mathrm{eV}$ corresponds to $T_{\text {eff }}=<54500 \mathrm{~K}$. It is impossible to estimate directly variations of the ultraviolet continuum of LL Cas, and this question remains open. At the same time our observations certify that $C(\mathrm{H} \beta)$ is changing with time. This may be the consequence of instability of the mass loss rate of the Mira star and increasing of the dust mass around the hot component. It is likely that dust and gas are mainly located in an orbit plane, thus a change of absorption does not affect the star's brightness if it is observed from a pole.

\section{References}

Allen, D. 1974, MNRAS, 168, 1

Belczynski, K., Mikolaejewska, J., Munari, U., et al. 2002, A\&AS, 146, 407 Huth, T. 1957, Verof. Astr. Rechen, 4, 10

Kharitonov, A. V., Tereshchenko V., \& Knyazeva, L. 1988, Spectrophotometric Catalog of stars, Nauka, Kazakhstan

Kondratyeva, L. 1992, AZh, 69, 1219 (Paper I)

Kornilov, V. G., Volkov, I. M., Zakharov, A. I., et al. 1991, Trudy Gosudarstvennogo Astron. Inst. Imeni Shternberga, 63, 3

Murset, U., \& Nussbaumer, H. 1994, A\&A, 282, 586

Perek, L., \& Kohoutek, L. 1967, Catalogue of galactic Planetary Nebulae, Prague 